\title{
Aneurysms of Two Sinuses of Valsalva with a Ventricular Septal Defect and Aortic Regurgitation
}

\author{
MAGDI H. YACOUB,* MARIO LISE, AND JOHN MUIR
}

From the National Heart Hospital, London W.1

The aortic sinuses of Valsalva, which represent three dilatations just above the aortic valve cusps, are largely intracardiac. Congenital aneurysms of one of the sinuses usually bulge into a cardiac chamber and eventually rupture causing an aortocardiac shunt which is a serious condition and can lead to death in a short time (Jones and Langley, 1949; Kwittken et al., 1965). Multiple aneurysms arising from different sinuses are rare.

In this paper a patient with two ruptured aneurysms of the right coronary and non-coronary sinuses of Valsalva, an infracristal ventricular septal defect, and aortic regurgitation is described. We believe that these lesions represent defects in the development of the bulbar septum, and are related to the syndrome of ventricular septal defect and aortic regurgitation (Nadas et al., 1964; Plauth et al., 1965; Van Praagh and McNamara, 1968).

\section{Case Report}

A man aged 37 was admitted to the National Heart Hospital for investigation after an attack of paroxysmal atrial fibrillation. A cardiac murmur had been first heard at the age of 6 at a routine medical examination, but he had remained symptom free until the onset of paroxysmal atrial fibrillation.

On examination, the pulse was collapsing in character, and the peripheral pulses were all palpable, with no delay. The blood pressure was $120 / 40 \mathrm{~mm}$. Hg. The cardiac impulse was markedly left ventricular in character, but the right ventricle was impalpable. At the lower end of the sternum both systolic and diastolic thrills could be felt. At the left sternal edge, there was a loud ejection systolic murmur that continued through a single second sound into early diastole. A third heart sound was heard in the mitral area. The venous pressure was not raised and there was no dependent oedema. The electrocardiogram confirmed the presence of sinus

* Present address: Department of Surgery, Section of Thoracic and Cardiovascular Surgery, University of Chicago, Chicago, Illinois 60637, U.S.A. rhythm, and the mean $Q R S$ vector in the frontal plane was plus $30^{\circ}$. There was evidence of left ventricular hypertrophy (SV2 $+\mathrm{RV} 5=82 \mathrm{~mm}$.). The chest $x$-ray showed moderate pulmonary plethora and slight cardiac enlargement, and calcium could be seen in the position of the aortic valve. The differential diagnosis before cardiac catheterization was a ventricular septal defect with aortic regurgitation or an aorto-pulmonary window. Right and left cardiac catheterization showed a gradient of $15 \mathrm{~mm}$. Hg across the outflow tract of the right ventricle, and left-to-right shunt at ventricular level. The pulmonary systemic flow ratio was $2 \cdot 7: 1$. A biplane cine-aortogram (Fig. 1) showed slight aortic regurgitation. The non-coronary sinus and cusp were dilated, and at the base of the cusp there was an aneurysm that appeared to extend down into the ventricular septum. Calcification was present at the base of the noncoronary sinus round the mouth of the aneurysm which appeared to have ruptured into the cavity of the right ventricle. A biplane Elema left ventricular angiogram showed a considerably dilated left ventricular cavity but no significant hypertrophy. No contrast medium could be seen in the right ventricular cavity before the aorta was filled. The angiograms were interpreted as showing an aneurysm of the non-coronary sinus, which had ruptured into the right ventricular cavity. There did not appear to be any evidence of a ventricular septal defect. Operation was performed, cardiopulmonary bypass was established, and the aorta was clamped and opened. On either side of the commissure between the right coronary and non-coronary cusps there was an orifice measuring about $7 \mathrm{~mm}$. in diameter and leading into an aneurysmal pouch; irregular calcification was also present in the same area (Fig. 2A). A transverse ventriculotomy was performed and the presence of two thin wall aneurysmal pouches, with multiple perforations bulging into the right ventricle, was confirmed (Fig. 2B). Repair of the aneurysms was performed by suturing from the aortic side and reinforcing the repair from the ventricular side. When the aortic clamp was relieved, it was apparent that gross aortic regurgitation was present. In addition, infracristal ventricular septal defect, measuring $6 \mathrm{~mm}$., was seen. The aorta was reopened, and careful inspec661 


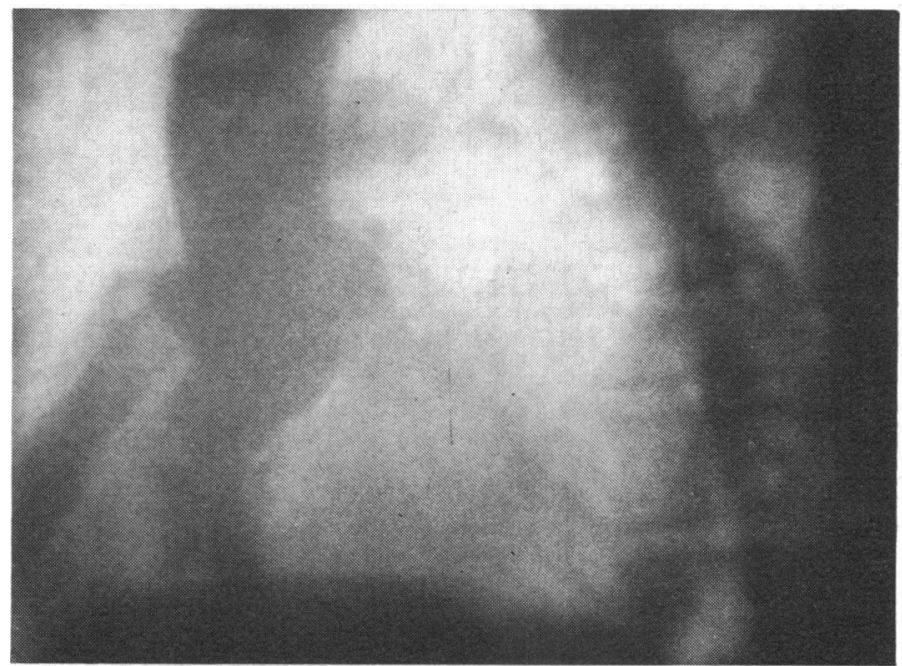

FIG. 1. - Aortogram showing mild to moderate aortic regurgitation as well as direct reflux of contrast medium from the aorta to the right ventricle.

a redundant prolapsing non-coronary cusp. The valve was excised and a homograft inserted. The heart failed to support the circulation even after a long period of left atriofemoral bypass. Necropsy revealed satisfactory repair of the ventricular septal defect and a competent homograft valve. However, there was extensive coronary atherosclerosis producing marked narrowing of all three arteries.

\section{Discussion}

Congenital aneurysm of a sinus of Valsalva was first described by Thurnam (1840). Jones and Langley (1949) reviewed the published material and found only 25 cases. An increasing number has been reported since (Lillehei, Stanley, and Varco, 1957; McGoon, Edwards, and Kirklin, 1958;

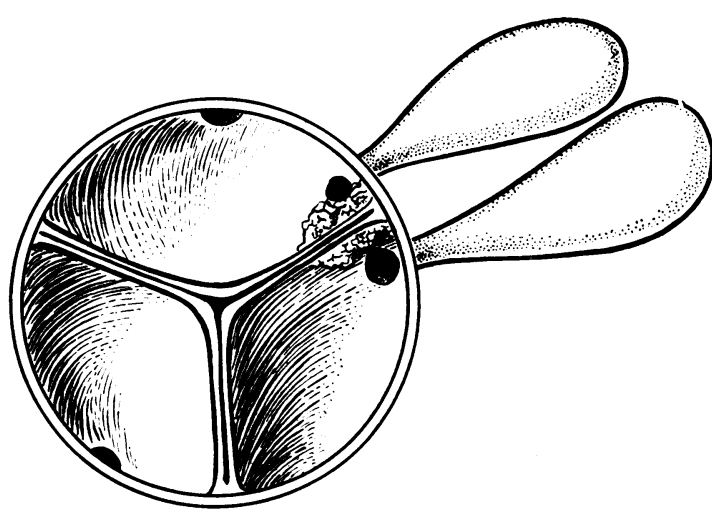

(A)

FIG. 2.-(A) Diagram showing the position of the openings into the aneurysms of the sinus of Valsalva. These were situated on either side of the commissure between the right and non-coronary cusps, and surrounded by calcification involving both cusps. (B) The aneurysmal sacs as seen from the right ventricular cavity. 
Gerbode et al., 1961; Magidson and Kay, 1963). Congenital aneurysm of a sinus of Valsalva is usually a long tubular structure with a narrow neck, and should not be confused with generalized dilatation of one or more of the sinuses. The pathological anatomy of the relations of the sinuses has been studied in detail by Edwards and Burchell (1957). The factors responsible for the development of a congenital aneurysm are not understood. The theories suggested include failure of the aortic media to join the annulus of the aortic valve (Edwards, Burchell, and Christensen, 1956), weakness of the annulus (Kwittken et al., 1965), cystic medial degeneration of the aorta (Steinberg and Geller, 1955), defect in the development of the elastic tissue at the base of the aorta (Venning, 1951; Heiner, Hara, and White, 1961), and defective development of the bulbar septum (Mall, 1912; Abbott, 1919; Jones and Langley, 1949). Several congenital anomalies have been described in association with ruptured aneurysm of the sinuses of Valsalva. These include ventricular septal defect (Jones and Langley, 1949; Brofman and Elder, 1957; Gerbode et al., 1961), malformations of the aortic valve (Herson and Symons, 1946), coarctation of the aorta (Dubilier, Taylor, and Steinberg, 1955), and pulmonary infundibular stenosis (Gerbode $e t$ al., 1961). In our patient we believe the abnormalities of the sinus of Valsalva, the aortic valve, and interventricular septum are all the result of a defect in the development of the bulbar septum. The syndrome of ventricular septal defect and aortic regurgitation (Nadas et al., 1964; Plauth et al., 1965) is probably a variant of this condition where the aortic valve and interventricular septum are affected. This is supported by the fact that many patients with this syndrome have anatomically abnormal aortic valves (Van Praagh and McNamara, 1968) rather than prolapse of one of the cusps produced by the presence of the ventricular septal defect (Nadas et al., 1964; Plauth et al., 1965).

In every patient with ruptured aneurysm of sinus of Valsalva, a deliberate attempt should be made to exclude the presence of ventricular septal defect or abnormality of the aortic valve.

\section{Summary}

A case of two aneurysms arising from the right coronary and non-coronary sinuses and rupturing into the right ventricle is described. In addition, the patient had aortic regurgitation and a ventricular septal defect. This combination is believed to represent a defect in the development of the bulbar septum, and is probably related to the syndrome of ventricular septal defect and aortic regurgitation.

\section{References}

Abbott, M. E. (1919). Clinical and developmental study of a case of ruptured aneurysm of the right anterior aortic sinus of Valsalva. In Contributions to Medical and Biological Research dedicated to Sir William Osler in Honour of his Seventieth Birthday, Vol. 2, p. 899. Hoeber, New York.

Brofman, B. L., and Elder, J. C. (1957). Cardioaortic fistula; temporary circulatory occlusion as an aid in diagnosis. Circulation, 16, 77.

Dubilier, W., Taylor, T. L., and Steinberg, I. (1955). Aortic sinus aneurysm associated with coarctation of the aorta. Amer. F. Roentgenol., 73, 10.

Edwards, J. E., and Burchell, H. B. (1957). The pathologic anatomy of deficiencies between the aortic root and the heart, including aortic sinus aneurysms. Thorax, 12, 125.

,$--\frac{1}{}$, and Christensen, N. A. (1956). Specimen exhibiting the essential lesion in aneurysm of the aortic sinus. Proc. Mayo Clin., 31, 407.

Gerbode, F., Osborn, J. J., Johnston, H. B., and Kerth, W. J. (1961). Ruptured aneurysms of the aortic sinuses of Valsalva. Amer. F. Surg., 102, 268.

Heiner, D. C. Hara, M., and White, H. J. (1961). Cardioaortic fistulas and aneurysms of sinus of Valsalva in infancy. A report of an aortic-left atrial communication indistinguishable from a ruptured aneurysm of the aortic sinus. Pediatrics, 27, 415.

Herson, R. N., and Symons, M. (1946). Ruptured congenital aneurysm of the posterior sinus of Valsalva. Brit. Heart f., 8, 125.

Jones, A. Morgan, and Langley, F. A. (1949). Aortic sinus aneurysms. Brit. Heart f., 11, 325.

Kwittken, J., Christopoulos, P., Dua, N. K., and Bruno, M. S. (1965). Congenital and acquired aortic sinus aneurysm. Arch. intern. Med., 115, 684.

Lillehei, C. W., Stanley, P., and Varco, R. L. (1957). Surgical treatment of ruptured aneurysms of the sinus of Valsalva. Ann. Surg., 146, 459.

McGoon, D. C., Edwards, J. E., and Kirklin, J. W. (1958). Surgical treatment of ruptured aneurysm of aortic sinus. Ann. Surg., 147, 387.

Magidson, O., and Kay, J. H. (1963). Ruptured aortic sinus aneurysms. Clinical and surgical aspects of seven cases. Amer. Heart F., 65, 597.

Mall, F. P. (1912). Aneurysm of the membranous septum projecting into the right atrium. Anat. Rec., 6, 291.

Nadas, A. S., Thilenius, O. G., LaFarge, C. G., and Hauck, A. J. (1964). Ventricular septal defect with aortic regurgitation; medical and pathologic aspects. Circulation, 29, 862.

Plauth, W. H., Braunwald, E., Rockoff, S. D., Mason, D. T., and Morrow, A. G. (1965). Ventricular septal defect and aortic regurgitation; clinical, hemodynamic and surgical considerations. Amer. F. Med., 39, 552.

Steinberg, I., and Geller, W. (1955). Aneurysmal dilatation of aortic sinuses in arachnodactyly: diagnosis during life in three cases. Ann. intern. Med., 43, 120.

Thurnam, J. (1840). On aneurisms, and especially spontaneous varicose aneurisms of the ascending aorta, and sinuses of Valsalva, with cases. Med.-chir. Trans., 23, 323.

Van Praagh, R., and McNamara, J. J. (1968). Anatomic types of ventricular septal defects with aortic insufficiency; diagnostic and surgical considerations. Amer. Heart F., 75, 604.

Venning, G. R. (1951). Aneurysms of sinuses of Valsalva. Amer. Heart f., 42, 57. 